Jap. J. Physiol., 27, 267-277, 1977

\title{
IMPAIRMENT OF ACQUISITION OF LEARNING BY INHIBITORS OF RNA OR PROTEIN SYNTHESIS
}

\author{
Nobuko Mishima and Toshiko Hashimoto \\ Department of Pharmacology, Tokushima Bunri University, \\ Tokushima, 770 Japan
}

\begin{abstract}
The effects of inhibitors of RNA or protein synthesis (cycloheximide, puromycin and actinomycin D) on acquisition of learning in mice were investigated using various types of shock avoidance tasks. Mice having uniform and superior learning ability were used as subjects. Drugs were injected into the lateral ventricle $1 \mathrm{hr}$ before training for avoidance tasks in three different shapes of maze and in a one-way shuttle box and for those based on light-dark discrimination. In all cases of training, the drugs were found to impair the acquisition of learning. The impairment was pronounced in a relatively complicated task such as $\mathrm{H}$-maze learning as compared to a simple task such as T-maze learning. The drugs never affected the performance of pretrained animals. During the discussion, the acquisition of learning would be related to some specific process of RNA and protein synthesis in the brain.
\end{abstract}

Up to now much effort has been made to discover the neurobiological processes that underlie learning and memory. Previous studies have indicated that cycloheximide, puromycin and actinomycin D impair the formation of long-term memory, but they do not affect the acquisition of learning or the formation of short-term memory (AgRANOFF et al., 1965; BARONDES and COHEN, 1967; FLEXNER et al., 1967; AgranofF, 1968; COHEN and BARONDES, 1968). These results suggest that the processes of synthesis of macromolecular substances such as protein and/or RNA in the brain do not mediate learning at least in its acquisition stage. However, SQUIRE et al. (1973) reported that a slight divergence of learning curves occurred between control mice and cycloheximide-injected ones at an advanced stage of training in a discrimination task, suggesting that the drug may affect the acquisition of learning.

Another approach to clarify the neurochemical mechanisms involved in learning and memory was to demonstrate changes in the level of macromolecular substances (Hyden and EgyHazI, 1962; Hyden and LANGE, 1965; AdAir et al.,

Received for publication January 24, 1977

三島宣子, 橋本淑子 
1968; Shashoua, 1968; SMith et al., 1975). AdAir et al. (1968) have shown that there is an increase of incorporation of labelled uridine into bulk RNA in the brains of those mice which were trained for avoidance conditioning. HydEN and LANGE (1970) have reported that the amount of an acidic protein called S-100 protein is increased in hippocampal nerve cells of rats after training for handtransfer tasks. However, some investigators who examined the effects of antimetabolites on learning insist that macromolecular synthesis is not required for the acquisition of learning. Thus, there seems to be contradiction in previous studies as to the role of protein synthesis in learning (AGRANOFF et al., 1965; Barondes and Cohen, 1967; Flexner et al., 1967; Agranoff, 1968; CoHen and BARONDES, 1968).

In the experiments described below, the effects of several inhibitors of RNA and protein synthesis on various types of learning were tested in lines of mice having homogeneous and superior maze learning ability. With all inhibitors tested, it was shown that although they did not affect the performance of previously learned tasks, they impaired the learning of new tasks and the degree of impairment increased as the dose increased.

\section{MATERIALS AND METHODS}

Subjects. DDY mice were purchased from the Awazu Company. From them those having relatively good learning ability were selected as the breeding subjects. Then, brother-sister breeding was repeated for four generations or more. Litters from such inbred mothers were divided into control and experimental groups to test the effects of drugs. The mice were used as experimental subjects when they were 30 to 35 days old and weighed 25 to $30 \mathrm{~g}$.

Maze training. Three shapes of maze, $\mathrm{T},+$ and $\mathrm{H}$, were used (Fig. 1). All had a stem and arms $30 \mathrm{~cm}$ in length and $5 \mathrm{~cm}$ in width. The stem had a start box at one end. To avoid side preference by an animal, the goal box was set so that an equal number of the subjects ran into goal boxes on the right and left sides. When a mouse did not run into the goal box within $5 \mathrm{sec}$ after opening the sliding door of the start box, then electric shocks of $50 \mathrm{~V}$ (AC) were delivered from the grid floor of the maze until the animal escaped into a goal box. The number of correct responses in a block of ten trials was expressed in percentage and taken as a score of learning.

Light-dark discrimination task. The apparatus used was the same T-maze as described above. All the running ways were darkened by covering the maze with black sheets, and either one of the goal boxes was lighted with a lamp $(40 \mathrm{~W})$. Mice were trained to run into the lighted goal box within $5 \mathrm{sec}$ after opening the door of the start box. The right and left goal boxes were lighted alternatively.

Shuttle box training. The conditioning box was divided into two compartments (each $15 \times 15 \times 15 \mathrm{~cm}$ ) by a center wall which had a small opening (Fig. 1). 
An animal was placed in either one of the compartments and conditioned to avoid electric shocks by running into the other compartment within $5 \mathrm{sec}$ after opened the door and a light was lit. Each training animal was always entered the same compartment at the start of each trial.

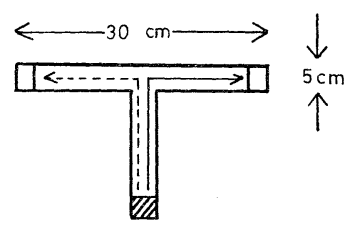

T-maze

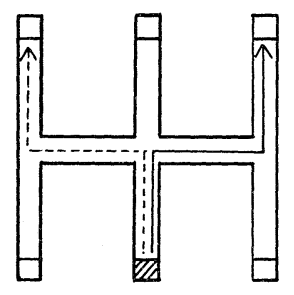

H-maze

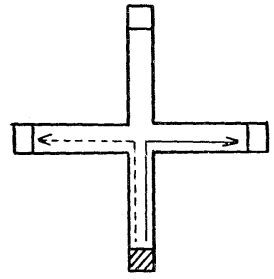

$+-m a z e$

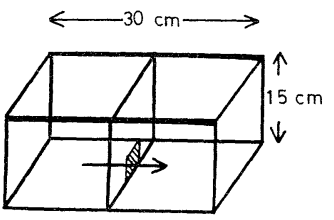

One way shuttle box

Fig. 1. Training apparatus. In the T-maze, +-maze and H-maze, the start box is indicated with hatches and the shortest running courses from the start to the goal have continuous and broken lines. The shuttle box for avoidance training consists of two compartments. The opening through which mice can move from one compartment to the other is indicated with hatches with an arrow.

Procedure of drug injection. Cycloheximide, puromycin, actinomycin D, cordycepin and its analogues were purchased from the Sigma Chemical Company. Each reagent was dissolved in physiological saline solution and $\mathrm{pH}$ of the solution was adjusted to neutral before use. After animals were lightly anesthesized with ether, the drugs were given by intraventricular injection. A syringe needle for the intraventricular injection was aimed at a point $1.0 \mathrm{~mm}$ lateral to the midsagittal plane, $4 \mathrm{~mm}$ anterior to the caudal suture of the parietal bone and $3 \mathrm{~mm}$ below the surface of the skull. The injection was made bilaterally, each side being injected an equal amount of drug. In what follows, the amount of administered drugs are given in total amount.

Statistics. Statistical comparisons of drug-injected group and control were made by the method of analysis of variance. 


\section{RESULTS}

\section{Selection of animals}

Ten DDY mice were trained in a T-maze. Two of them reached a level of performance higher than $80 \%$ and five reached a level of 50 to $70 \%$, whereas three remained in the level of $20 \%$ even after 40 trials (Fig. 2a). Thus, commercially available DDY mice were extremely heterogenous in learning in the T-maze. The variability in learning ability was not constant among different lots of purchased animals. Therefore, we tried to obtain mice having uniform and

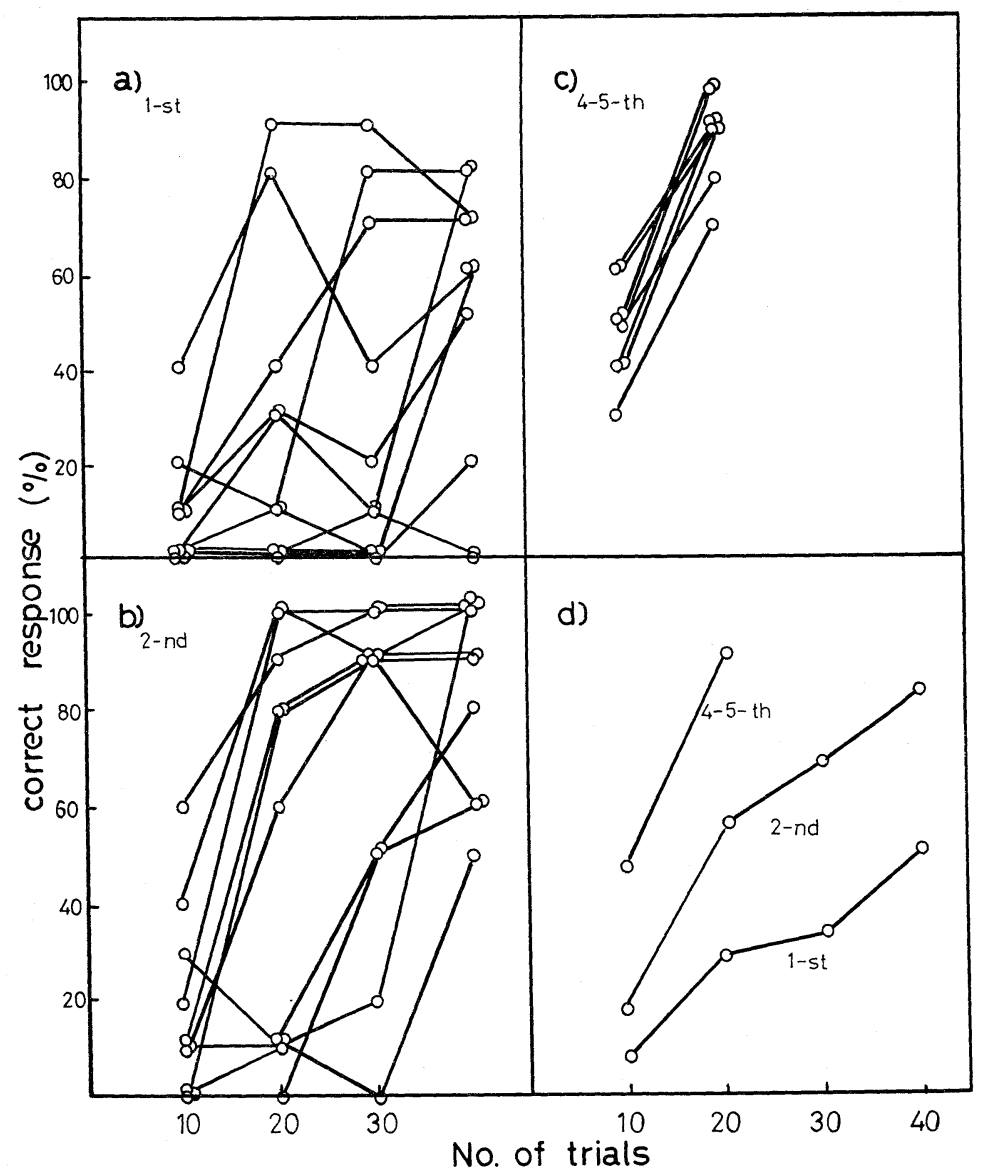

Fig. 2. Learning curves of mice of various generations obtained by brother-sister mating. Each curve in $a, b$, and c represents the performance of individual DDY mice in T-maze learning consisting of 30-40 trials. a, 10 mice obtained commercially (1st generation). b, 10 mice of the 2 nd generation. c, 8 mice of the 4-5th generations. d, average learning curves for different generations. 
superior maze learning ability. The mice exhibiting a final score higher than $80 \%$ were selected and allowed to breed in brother-sister mating. In the second generation, about half of the subjects showed similar learning curves, characterized by a rapid increase in the score (Fig. 2b). However, some heterogeneity of the learning ability still remained in these subjects. From these subjects, we selected mice with high learning ability and brother-sister breeding were continued. Most of the fourth to fifth generation offsprings reached a level of more than $80 \%$ in only 20 trials (Fig. 2c). This population had a relatively homogeneous ability for T-maze training, but did not have uniform ability in more difficult tasks, such as H-maze training.

The mean learning curves of T-maze training in mice of the first, second and fourth to fifth generation are plotted in Fig. 2d. Essentially, similar learning curves were obtained with the offsprings born from a given pair of parents. But, different litter mates, though obtained after a repetition of the inbreeding, usually showed different learning abilities. Then, appropriate types of mazes were chosen for training according to the learning ability of the animals. The offsprings whose siblings showed a relatively high learning ability were used for more complicated tasks, such as the + or H-mazes. None of the subjects were pretrained.

\section{Effects of cycloheximide and puromycin}

Drugs dissolved in saline were injected into the lateral ventricle. One hour after the injection the animals, learning ability was tested in the + -maze, the shuttle box or the light-dark discrimination task. An equal volume of saline solution $(10 \mu \mathrm{l})$ was injected into the control groups. In all of the three different training experiments, the learning ability was remarkably low when mice received $300 \mu \mathrm{g}$ of cycloheximide. The placebo group acquired a score of more than $80 \%$ in 30 to 40 trials (Fig. 3a). In the drug-injected group, the score of + -maze learning remained only $20 \%$ after 40 trials [Fig. 3a; $F(1,18)=20.5, p<0.001$ ]. Other types of learning were also severely affected by the injection [shuttle box in Fig. $3 \mathrm{~b} ; F(1,6)=8.6, p<0.05$ : light-dark discrimination task in Fig. $3 \mathrm{c} ; F(1,14)$ $=13.2, p<0.01]$. Figure $3 \mathrm{c}$ shows that the inhibition was more severe as the dose of the drug was increased.

Puromycin, a potent inhibitor of protein synthesis, was also used in tests in the T-maze and the H-maze. In either case of maze training, it was observed that the antibiotic caused a decline in the correct response: in the T-maze task the learning of the puromycin-injected mice was also extensively inhibited [Fig. $4 \mathrm{a} ; F(1,10)=$ 8.8, $p<0.05$ ] and in the H-maze task the drug-injected mice did not show any significant learning even after 30 trials [Fig. $4 b ; F(1,5)=115.5, p<0.001$ ]. In the cases of more complicated training, the effect of the antibiotic was greater than those in relatively simple maze tasks. 


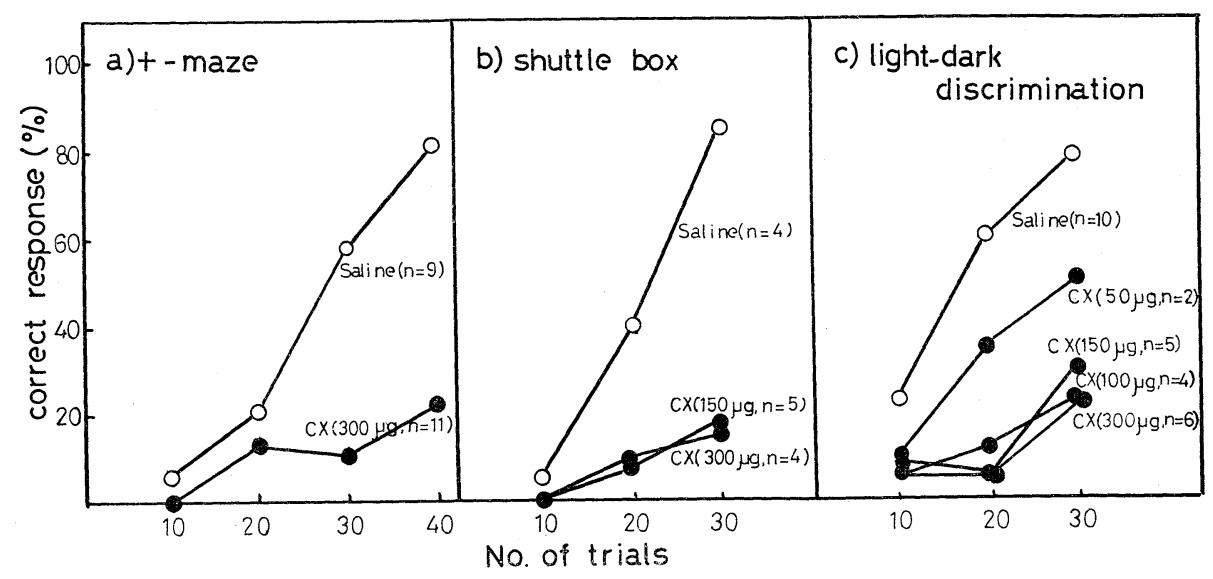

Fig. 3. Learning curves of mice injected with saline and cycloheximide (CX). a, +-maze learning. $b$, shuttle box learning. c, light-dark discrimination learning. The drug and saline were given intraventricularly $1 \mathrm{hr}$ before the start of training.

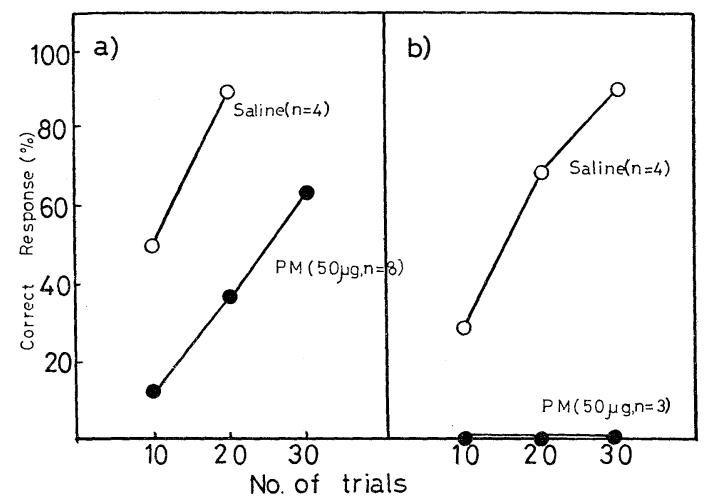

Fig. 4. Learning curves of mice injected with saline and puromycine (PM). a, T-maze learning. $\mathrm{b}, \mathrm{H}$-maze learning. The drug and saline were given intraventricularly $1 \mathrm{hr}$ before the start of training.

\section{Effects of actinomycin $D$ and cordycepin}

Actinomycin D is a potent inhibitor of transcription and cordycepin (3deoxy-adenosine) is an inhibitor of polyadenylation of m-RNA. Their effects on the acquisition of the light-dark discrimination task were tested. As presented in Fig. 5a, the effect of actinomycin D was only slight at a dose of $50 \mu \mathrm{g} / \mathrm{mouse}$. Because of low solubility of actinomycin D, it was impossible to use more than $50 \mu \mathrm{g}$. At a dose of $45 \mu \mathrm{g}$ of cordycepin the inhibition of learning was significant [Fig. 5b; $F(1,22)=14.0, p<0.01]$. A dose of $70 \mu \mathrm{g}$ impaired the learning more severely [Fig. $5 b ; F(1,32)=20.0, p<0.001]$. When analogues such as adenosine and 2-deoxyadenosine were tested at a dose of $70 \mu \mathrm{g}$, these did not show any 
significant effect on learning (Fig. 5b, $F<1$ ). Thus, the inhibitors of RNA and protein synthesis impaired performance in several kinds of training tasks, suggesting that these macromolecular substances may have a role in the neurochemical process to establish learning.

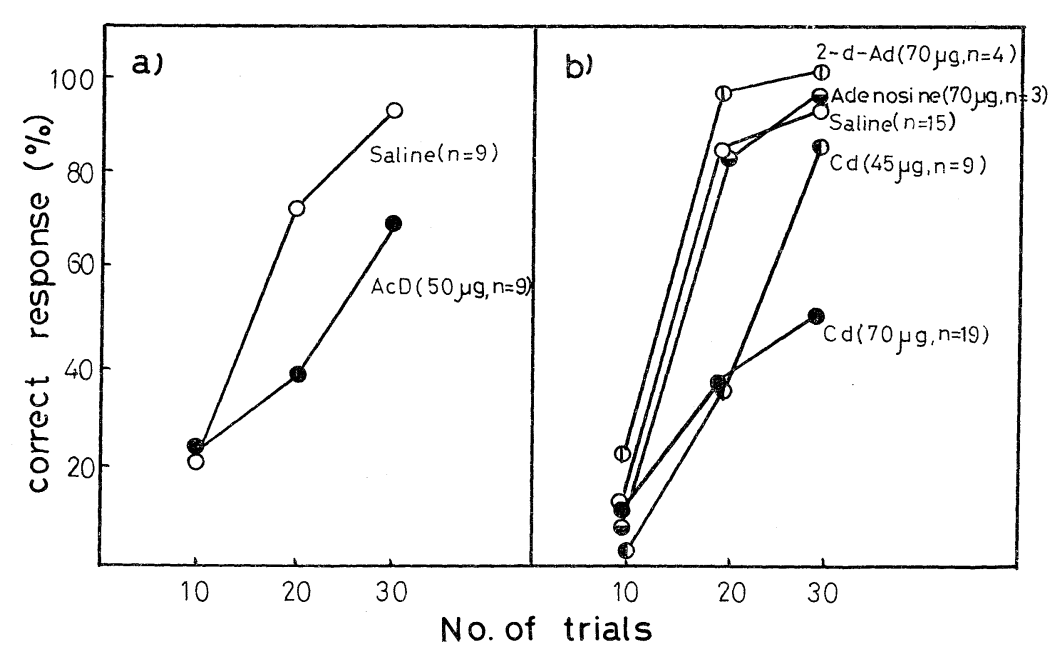

Fig. 5. Effects of actinomycine D (AcD), cordycepin (Cd), adenosine (Ad) and 2-deoxyadenosine (2-d-Ad) upon light-dark discrimination learning. The drugs and saline were injected bilaterally into the ventricles $1 \mathrm{hr}$ before the start of training.

\section{Effects of drugs on established task}

It may be possible that the above-mentioned effects of drugs are attributable to impairment of general cellular activities. However, this possibility was ruled out by the following experiment. The offsprings of the fourth to fifth generation could retain about the same performance level as that seen at the end of the first training for more than several months. Cycloheximide or puromycin was injected into the ventricle of such pretrained animals. First, the mice were given 40 trials. Then, one day after this training session, the drug was injected, and one hour thereafter ten more trials were run. As shown in Fig. 6, the task performance was not significantly affected by the drugs at doses sufficient to affect learning acquisition (see filled circles in Fig. 6). Sometimes, puromycin induced inhibition of performing the established task, but this only happened when much higher doses $(100 \mu \mathrm{g})$ were used. It is concluded that the effects of the drugs on learning acquisition so far mentioned were obtained with a range of doses which do not impair the performance of once established task.

From the above observation, it can be suggested that blocking agents of protein and RNA synthesis may act on some specific steps of the neurochemical reaction to establish learning. 


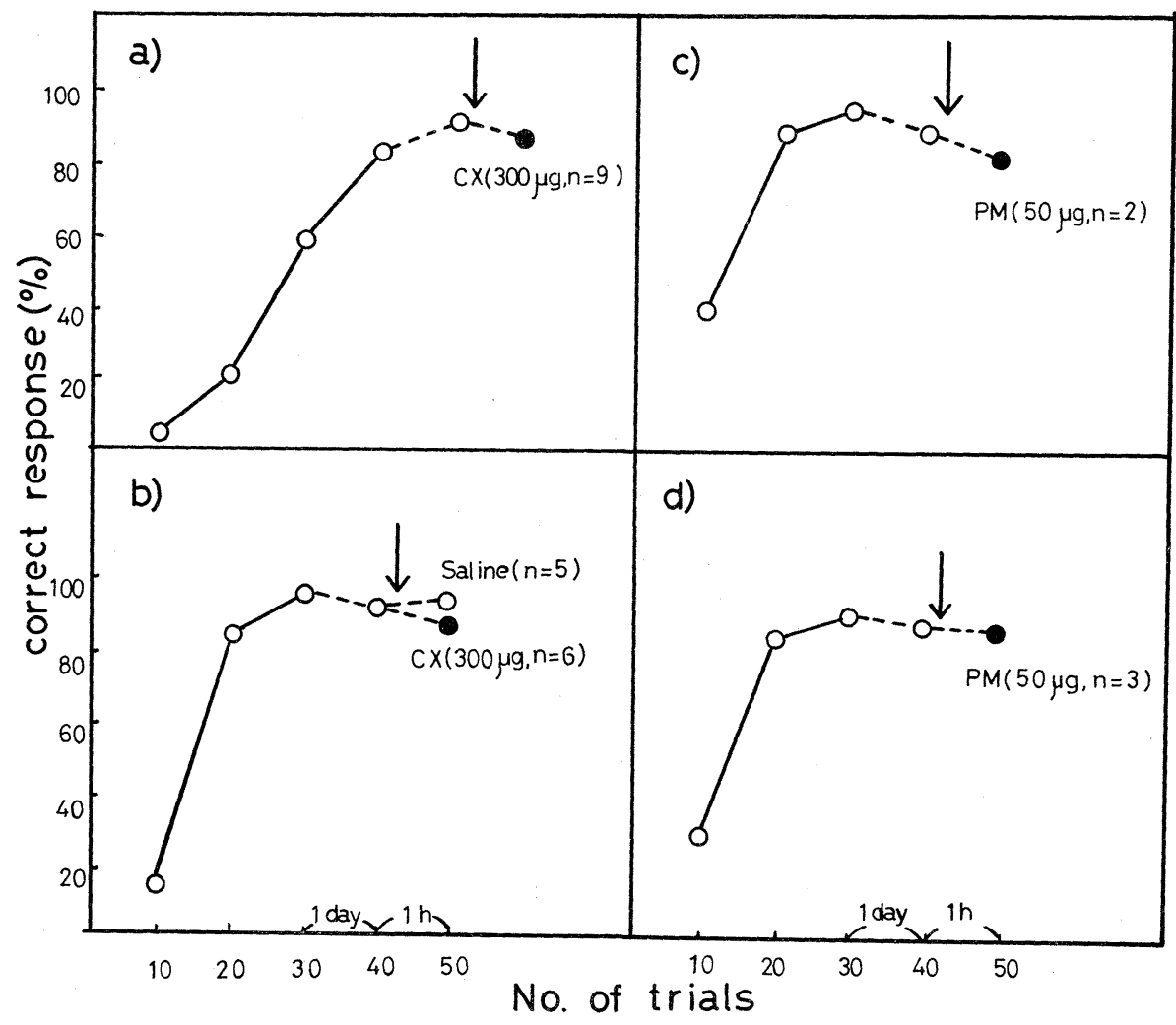

Fig. 6. Effects of antibiotics on performance of previously learned tasks. One day after the initial training (30-40 trials) retention tests of 10 trials were made and followed by intraventricular injection of cycloheximide (CX) and puromycine (PM). One hour thereafter mice were tested for more ten trials. a, +-maze training. b, H-maze training. c, T-maze training. d, H-maze training.

\section{Comparison of routes of drug administration}

In the following experiments we examined whether the effects of cycloheximide were different according to the method of administration. Since the data presented in the previous sections were obtained by the method of intraventricular injection, we tried to examine the effects of cycloheximide administered by intracerebral and intraperitoneal injections. After the animals were anesthetized with ether, the intracerebral injection was made bilaterally at a site of the brain, $2 \mathrm{~mm}$ lateral to the midsagittal plane, $2 \mathrm{~mm}$ anterior to the caudal suture of the parietal bone and $3 \mathrm{~mm}$ below the surface of the skull. Ten $\mu \mathrm{l}$ solution containing cycloheximide of $300 \mu \mathrm{g}$ was used for the intracerebral injection. Six mirigrams of the same drug was given intraperitoneally as a $0.2 \mathrm{ml}$ saline solution. In both cases the +-maze training was employed. The effect of intracerebral cycloheximide is shown in Fig. 7c; the inhibition of learning was considerably weaker as 
compared with that obtained by intraventricular cycloheximide. The latter is seen in Fig. 7a in which the data of Fig. 3a are redrawn. By the intraperitoneal injection the learning was slightly affected though the administered dose was as high as $6 \mathrm{mg}$ [Fig. $7 \mathrm{~b} ; F(1,6)=0.45, p>0.05]$. These results indicate that ventricular injection of the drug is more effective than the other two ways of administration. We recently found that inhibition of learning of learning by intrahippocampal injection is as effective as observed by ventricular injection.

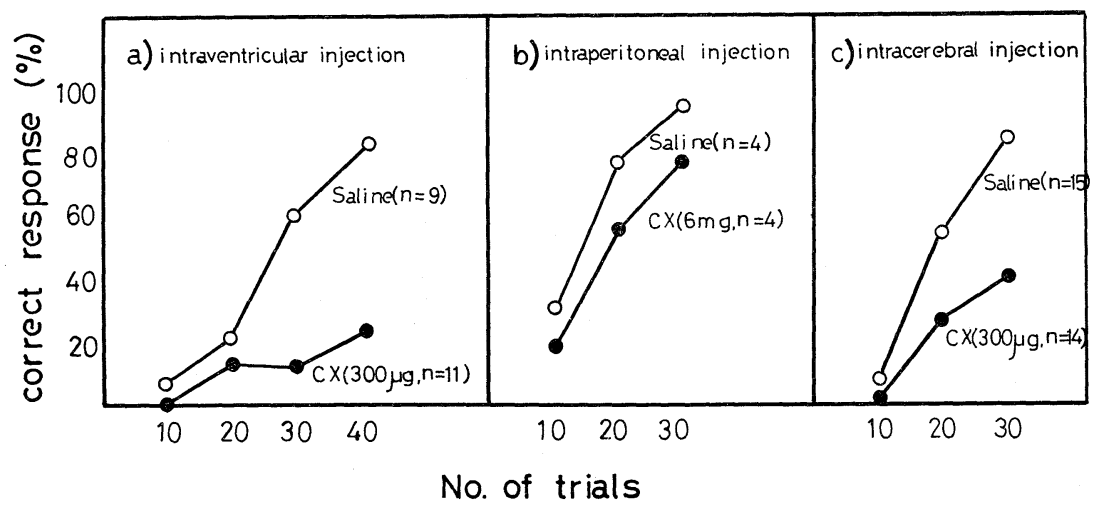

Fig. 7. Effects upon t-maze learning of cycloheximide given in three different routes. a, intraventricular injection. The data are the same as in Fig. 3a. b, intraperitoneal injection. c, intracerebral injection. In each case, the training was started $1 \mathrm{hr}$ after the drug administration.

\section{DISCUSSION}

Learning, or memory, in animals is one of the most complicated of biological phenomena. In order to deal with it quantitatively, simplification of the experimental design and suppression of fluctuating factors among subjects is crucial. The reduction of individual variations due to genetic factors seems to be very important. Previous investigators have indicated that differences in genotype are associated with differences in maze learning ability (BOVET et al., 1969). In the present experiment, we examined the drug effect on homogeneous experimental subjects prepared by inbreeding. Animals having superior learning ability were selected and bred by brother-sister mating. A relatively homogeneous population of mice with a high learning ability for the T-maze was obtained after the inbreeding had been repeated 4 to 5 times (Fig. 2c). Since these animals were not highly inbred, there still remained a divergence in the ability for learning tasks among different litter mates. We overcame such drawbacks by taking the mice from the same venter as control and an experimental group. By this procedure, we obtained very reliable results in which the inhibitors of RNA and protein synthesis can certainly affect learning acquisition. 
In testing the effect of an inhibitor on training animals, the drug must be administered so as to maintain sufficient concentration throughout the training duration. Therefore, a short learning time is preferable when we wish to block cerebral protein synthesis with a minimum impairment of general physiological functions. Accordingly, we selected superior animals capable of reaching high performance levels in a small number of trials. The offsprings obtained after a repetition of selective breeding not only showed uniform learning ability, but also could finish the training task faster (Fig. 2). These animals were able to finish each training task within 30 to $60 \mathrm{~min}$.

BARONDES and COHEN (1967) have reported that subcutaneously administered cycloheximide $(120 \mathrm{mg} / \mathrm{kg})$ did not cause a reduction in the light-dark discrimination task of mice. We observed in the present experiments that the same amount of cycloheximide, when not applied via the cerebral ventricle, did not affect maze learning. When it was given intraperitoneally, even a dose as high as $240 \mathrm{mg} / \mathrm{kg}$ caused only a slight reduction in learning (Fig. 7b). When the drug bilateraly injected into the lateral ventricle in a dose of $12 \mathrm{mg} / \mathrm{kg}$, the task could hardly be established (Fig. 7a). But, when injected directly into the cerebral tissue, the same amount of drug was not so effective as the intraventricular injection (Fig. 7c). Therefore, it can be said that the dose as well as the route of drug administration influence considerably the inhibitory effect.

Some damage of brain structure due to intracerebral injection might be responsible for the inhibition of learning. But, this is not case because intracerebral injection of saline by the same method did not cause any decline in learning. Alternatively, impairment of fundamental functions of the brain caused by intracerebral injection of the drug could result in inhibition of learning.

Cordycepin (3-deoxy-adenosine) which is known as a blocking agent of polyadenylation of m-RNA showed a marked inhibition in learning. In contrast, administration of a similar amount of its analogues, adenosine and 2-deoxyadenosine, did not cause any significant inhibitory effect. Such selective inhibitory effect on learning due to specific drugs indicate that the impairment of the learning is not due to a general physiological disturbance, but is related to changes in specific processes of macromolecular synthesis.

Recently, increased protein and RNA synthesis were demonstrated as occuring as neurochemical changes during learning (HYDEN and LANGE, 1965; ZEMP et al., 1966; AdAIR et al., 1968; SмITH et al., 1975). A question has arisen whether macromolecular substances synthesized during learning may play a role in the process of establishment of learning and/or in its later stage of memory storage. Present findings favour the view that the newly synthesized macromolecules are necessary for the processes of learning acquisition and that both RNA and protein synthesis are involved in establishment of learning as well as formation of long memory. 
The authors wish to thank Drs. K. Iwama and F. Imamoto, Osaka University, for their helpful suggestions during the preparation of the manuscript.

\section{REFERENCES}

Adair, L., Wilson, J. E., Zemp, J. W., and Glassman, E. (1968) Brain function and macromolecules, III. Uridine incorporation into polysomes of mouse brain during short-term avoidance conditioning. Proc. Natl. Acad. Sci. USA, 61: 606-613.

Agranoff, B. W., Davis, R. E., and Brinck, J. J. (1965) Memory fixation in the goldfish. Proc. Natl. Acad. Sci. USA, 54: 788-793.

AgranofF, B. W. (1968) Actinomycin D blocks formation of memory of shock avoidance in goldfish. Science, 158: 1600-1601.

Barondes, S. H. and Cohen, H. D. (1967) Delayed and sustained effect of acetoxycycloheximide on memory in mice. Proc. Natl. Acad. Sci. USA, 58: 157-164.

Bovet, D., Bovet-Nitti, F., and Oliverio, A. (1969) Genetic aspects of learning and memory in mice. Science, 163: 139-149.

Cohen, H. D. and BARondes, S. H. (1968) Acetoxycycloheximide effect on learning and memory of light-dark discrimination. Nature, 218: 271-273.

Flexner, L. B., Flexner, J. B., and Robert, R. B. (1967) Memory in mice analized with antibiotics. Science, 155: 1377-1383.

Hyden, H. and EgyhazI, E. (1962) Nuclear RNA changes in nerve cells during learning experiments in rats. Proc. Natl. Acad. Sci. USA, 48: 1366-1373.

HYDEN, H. and LANGE, P. W. (1965) A differentiation of RNA response in neurons early and late during learning. Proc. Natl. Acad. Sci. USA, 53: 946-952.

Hyden, H. and LANge, P. W. (1970) Correlation of the S-100 protein with behaviour. Exp. Cell Res., 62: 125-132.

SHASHOUA, V. E. (1968) RNA changes in goldfish brain during learning. Nature, 217: 238-240.

Smith, J. E., Heistad, G. T., and Thompson, T. (1975) Uptake of ${ }^{3} \mathbf{H}$-uridine into brain and incorporation into brain RNA of rats exposed to various training tasks-a biochemical analysis. Pharm. Biochem. Behav., 3: 447-454.

Squire, L. R., Smith G. A., and Barondes, S. H. (1973) Cycloheximide affects memory within minutes after the onset of training. Nature, 242: 201-202.

Zemp, J. W., Wilson, J. E., Schlesinger, K., Boggan, W. O., and Glassman, E. (1966) Brain function and macromolecules I, Incorporation of uridine into RNA of mouse brain during short-term training experience. Proc. Natl. Acad. Sci. USA, 55: 1423-1431. 